\title{
Outcome Measures in Bariatric Surgery
}

\author{
Kamal K. Mahawar ${ }^{1}$
}

Published online: 16 August 2015

(C) Springer Science+Business Media New York 2015

\section{Dear Editor,}

One must congratulate Brethauer and co-authors on their painstaking attempt to standardise reporting outcomes in bariatric and metabolic surgery [1]. Inconsistencies with reporting outcomes have been a longstanding problem with bariatric and metabolic surgery [2], and if properly followed, these guidelines will make future systematic analysis of bariatric literature easier and more meaningful.

There are two other important aspects of outcomes reporting with bariatric surgery that this article does not however address. The first aspect is insistence by some reviewers that every study report on as many of these outcome measures as possible. Obesity is a multisystem disease and bariatric surgery has effect on a large number of organs and systems. Researchers in bariatric surgery often face the problem of too many outcome measures, and some authors have argued for developing a set of core outcome standards [3, 4]. Reporting on even a limited number of outcomes to the standards of these guidelines [1] may prove difficult outside of large funded trials. We hence suggest that editors and reviewers not only enforce these guidelines but also allow researchers to focus on any number of outcomes. In this context, reporting on even one outcome may be satisfactory if carried out properly.

The second problem that we face while reporting outcomes is that our reporting methods are an attempt to convert continuous variables like excess weight exposure or exposure to high blood sugar or high blood pressure into discrete variables like excess weight, blood sugar, and blood pressure. Though

Kamal K. Mahawar

kamal_mahawar@hotmail.com

1 Bariatric Unit, Sunderland Royal Hospital, Sunderland SR4 7TP, UK values at salient time points are important, they are merely snapshots and do not represent the full picture. We have so far not seen any attempt to talk about area under the curve for these variables, which will probably be a more meaningful way to compare them. For example, it is conceivable that gastric band and gastric bypass result in same excess weight loss at 10-year follow-up but one suspects they will have a different area under the curve. Reporting on population exposure rather than prevalence at different time points will recognise the chronic nature of obesity and its associated co-morbidities. It will however not be easy to develop these measures and will probably need some help from our statistician friends.

Conflict of Interest The author declare that there are no competing interests.

Statement on Human and Animal Rights This article does not contain any studies with human participants or animals performed by any of the authors.

\section{References}

1. Brethauer SA, Kim J, El Chaar M, Papasavas P, Eisenberg D, Rogers A, et al. ASMBS clinical issues committee. Standardized outcomes reporting in metabolic and bariatric surgery. Obes Surg. 2015;25(4): 587-606.

2. Dixon JB, McPhail T, O’Brien PE. Minimal reporting requirements for weight loss: current methods not ideal. Obes Surg. 2005;15(7): 1034-9.

3. Hopkins JC, Howes N, Chalmers K, Savovic J, Whale K, Coulman $\mathrm{KD}$, et al. By-band trial management group. Outcome reporting in bariatric surgery: an in-depth analysis to inform the development of a core outcome set, the BARIACT study. Obes Rev. 2015;16(1): 88-106.

4. Macefield RC, Jacobs M, Korfage IJ, Nicklin J, Whistance RN, Brookes ST, et al. Developing core outcomes sets: methods for identifying and including patient-reported outcomes (PROs). Trials. 2014;15:49. 